\title{
Electrochemical Sensing and Consumption of Intracellular NADH in Mammalian Cells for their Applications
}

\author{
Hiroaki Shinohara ${ }^{1}$, Hiroto Taketsu ${ }^{2}$, Masato Kyuji ${ }^{2}$ and Minoru suga ${ }^{1}$ \\ ${ }^{1}$ Graduate School of Science and Engineering for Research, University of Toyama, Japan \\ 2 Graduate School of Science and Engineering for Education, University of Toyama, Japan \\ e-mail: hshinoha@eng.u-toyama.ac.jp
}

\begin{abstract}
:
Electrochemical sensing of intracelluar NADH was performed with 1-Methoxy-PMS as a cell membrane-permeable oxidizing mediator to monitor viability and number of mammalian cells. The electrochemical sensing for intracellular NADH was applied to evaluate the inhibition effect of oxamate to intracelluar lactate dehydrogenase (LDH). Futhermore, electrochemical consumpsion of intracelluar $\mathrm{NADH}$ to induce cell death also examined on the cell-adhered trasparent electrode by constant oxidizing voltage application with 1-Methoxy-PMS as a redox cycling mediator.
\end{abstract}

Key words: Intracellular NADH, Electrochemical sensing, Viable activity, Electrochemical consumption, Metabolic reaction

\section{Introduction}

$\mathrm{NADH}$ is one of the most important compounds to promote many enzymatic reactions and ATP production in cytosol and mitochondria. Then, it is very effective to monitor the intracellular $\mathrm{NADH}$ for mammalian cell viability assay. Recently we can use colorimetric assay using the change of water soluble tetrazolium to formazan by 1-Methoxy-5-methylphenazinium methylsulfate, a cell membrane permeable mediator reduced with $\mathrm{NADH}$ to monitor intracellular $\mathrm{NADH}$ and to measure the number of cells. Electrochemical methods for monitoring the intracellular NADH with other electron mediators have been also studied since before. But the application of 1-Methoxy-5-methylphenazinium methylsulfate (abbreviated to MMP) to electrochemical sensing of intracellular NADH has not been reported.

Here we report the electrochemical sensing of intracellular NADH with MMP and applications for cell counting and evaluation of the inhibition effect of oxalate to intracellular lactate dehydrogenase. Furthermore, we thought the electrochemical recycling of MMP was available to consume the intracellular $\mathrm{NADH}$ and to induce cell death. Cell killing by continuous electrochemical oxidation of MMP was examined by constant voltage application onto the cell-adhered transparent electrode.

\section{Experimental Method}

PC12 (Rat adrenal pheochromocytoma) cell was used for all experiments. The cell was cultured in a DMEM supplemented with $5 \%$ FBS and $10 \%$ HS. The Electrochemical measurement chamber was prepared by attaching a flexible wall made from silicon resin on a screen printed dual carbon electrode with an $\mathrm{Ag} / \mathrm{AgCl}$ reference electrode. The cell suspended medium was injected into the electrochemical chamber. After $10 \mathrm{~min}$ from the cell seeding, MMP was added into the chamber at the final concentration of $500 \mu \mathrm{M}$ and then electrode potential was applied at $0.1 \mathrm{~V}$. The toxic effect of oxamate was evaluated by coaddition with MMP into the cell-suspension.

\section{Result and Discussion}

Our experimental data demonstrated that the oxidation current of MMP at 5 min from the $0.1 \mathrm{~V}$ application depended on the number of cells in the chamber and the toxic effect of oxamate could be evaluated by this oxidation current decrease. It was considered that the current decrease was due to the inhibition of NADH production by LDH in the cell with oxamate. Cell killing by continuous electrochemical oxidation of MMP will be presented in the conference.

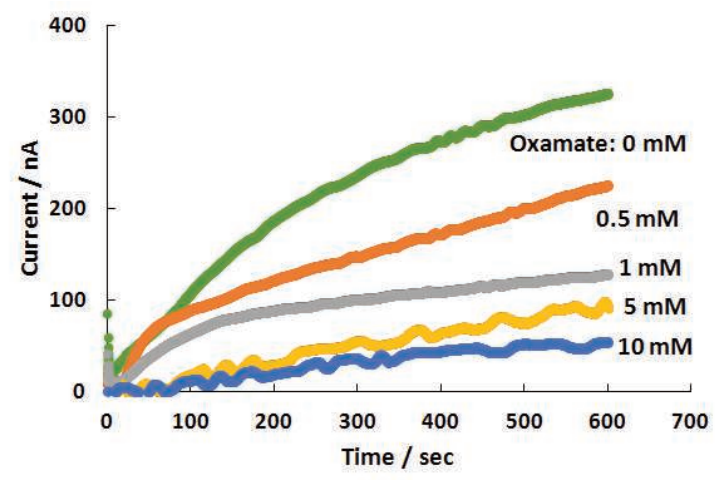

Fig. 1. Electrochemical sensing of intracellular $\mathrm{NADH}$ by chronoamperometry under $0.1 \mathrm{~V}$ vs.AgAgCl application in the presence of various concentration of oxamate. 